\title{
Path in directed graph with time duration at each vertex
}

\author{
Thinh D. Nguyen
}

\section{Introduction}

In this section, we give the definition of our problem.

\section{Given}

- a directed graph $G=(V, E, d)$, where $d: V \rightarrow I\left(Q_{0}^{+} \cup\{+\infty\}\right)$ (here $Q_{0}^{+}$denotes the set of nonnegative rational numbers and $I\left(Q_{0}^{+} \cup\{+\infty\}\right)$ the set of intervals, bounded or unbounded above, with non-negative rational bounds) is a function associating with each vertex $v \in V$ an interval $d(v)=[a, b]$ for some $a \in Q_{0}^{+}, b \in Q_{0}^{+} \cup\{+\infty\}$ and $a \leq b$,

- two vertices $s, t \in V$ and

- $\quad$ an integer $h$ encoded in binary,

We have to decide whether or not there exist

- a path in $G$, possibly with repeated vertices and edges, $\left(v_{0}, v_{1}, \cdots v_{n-1}, v_{n}\right)$, with $v_{0}=s$ and $v_{n}=t$ and

- a list of values $d_{0}, \ldots, d_{n} \in Q_{0}^{+}$, such that $\sum_{i=0}^{n} d_{i}=h$ and for all $i=0, \ldots, n, d_{i} \in d\left(v_{i}\right)$.

Intuitively, we have to find a path in $G$, possibly where we get to the same vertices/edges also more than once, and where we remain in each vertex for a non-negative rational amount of time allowed by the minimum/maximum duration function, such that the overall time of the path equals $h$.

This can be solved easily in PSPACE. We want to show that it is in NP (we already know it is NP-hard). This is not trivial to prove, as we may have $h \in \Theta\left(2^{n}\right)$, for instance. Thus the required path may have length exponential in both $|V|$ and in the binary encoding of $h$.

\section{Hardness of our problem}

If zero-width durations are allowed, then the PARTITION PROBLEM can be reduced to our problem: Order the input numbers $x_{i}$ arbitrarily. Make a path with a vertex for each number $x_{i}$, with zero-width interval $\left[x_{i}, x_{i}\right]$. Prepend $s$ to this path, and append $t$. Add edges directed from every lower-numbered vertex to every higher-numbered others, and set $h=t / 2$ where $t$ is the sum of all input numbers.

\section{Polynomial-time verifiable certificate}

This solution is by Gerhard Woeginger.

In order to prove that this problem belongs to NP, we provide a polynomial-size certificate, and then we show how to check it in deterministic polynomial time. 
The certificate is just the following: a set of integers $\left\{x_{u, v} \mid(u, v) \in E\right\}$. Intuitively, $x_{u, v}$ is the number of times the solution path traverses $(u, v)$.

We now describe the verification algorithm.

1. We consider the subset $E^{\prime}$ of edges of $G, E^{\prime}:=\left\{(u, v) \in E \mid x_{\mathrm{u}, \mathrm{v}}>0\right\}$. We check whether $E^{\prime}$ induces a strongly (undirected) connected subgraph of $G$.

2. We check whether

- $\quad \sum_{(\mathrm{u}, \mathrm{v}) \in \mathrm{E},} x_{u, v}=\sum_{(\mathrm{v}, \mathrm{w}) \in \mathrm{E},} x_{v, w}$, for all $v \in V \backslash\{s, t\}$;

- $\sum_{(u, s) \in \mathrm{E},} x_{u, s}=\sum_{(s, w) \in \mathrm{E}}, x_{s, w}-1$

- $\sum_{(u, t) \in \mathrm{E},} x_{u, t}=\sum_{(t, w) \in \mathrm{E}}, x_{t, w}+1$.

3. For all $v \in V \backslash\{s\}$, we define $y_{v}:=\sum_{(\mathrm{u}, \mathrm{v}) \in \mathrm{E}}{ }^{\prime} x_{u, v}$, i.e., the number of times the solution path gets into $v$. Moreover, $y_{s}:=\sum_{(s, u) \in E}, x_{s, u}$.

4. We check whether there exist real values $z_{v}$, for every $v \in V$, such that

- $d_{\max }(v) y_{v} \leq z_{v} \leq d_{\max }(v) y_{v}$ (here $d_{\min }$ and $d_{\max }$ denote resp. the lower and the upper bound of the rational interval $d(v)$, and

- $\sum_{v \in V} Z_{v}=h$.

We now sketch the correctness:

Steps 1. and 2. together check that the values $x_{u, v}$ for the arcs specify a directed Eulerian path from $s$ to $t$ (we refer to [1, section 1.3])

Steps 3. and 4. calculate $z_{v}$, for all $v \in V$, which is the total waiting time of the path on the node $v$. We observe that the (in)equalities of step 4 . form a linear program (LP), which can be solved in deterministic polynomial time (e.g., using the ellipsoid algorithm).

\section{References}

[1] Dieter Jungnickel, Graphs, Networks and Algorithms, 3rd edition, 2008 